\title{
Characterization of Countably Normed Nuclear Spaces
}

\author{
G.K.Palei ${ }^{1} \&$ Abhik Singh ${ }^{2}$ \\ 1. Department of Mathematics, B. N. College, Patna University, Patna-800005 (INDIA) \\ 2. Research Scholar, Patna University, Patna-800005 (INDIA).
}

\begin{abstract}
Every count ably normed nuclear space is isomorphic to a subspace of a nuclear Frechet space with basis and a continuous norm. The proof as given in section 2 is a modification of the Komura-Komura inbedding theorem .In this paper, we shall show that a nuclear Frechet space with a continuous norm is isomorphic to a subspace of a nuclear Frechet space with basis and a continuous norm if and only if it is countably normed.The concept of countably normedness is very important in constructing the examples of a nuclear Frechet space. Moreover, the space with basis can be chosen to be a quotient of $(s)$.
\end{abstract}

Key words and phrases: Nuclear frechet space, Countably normed and Nuclear Kothe space.

\section{Normed Spaces:}

Let $\mathrm{E}$ be a Frechet space which admits a continuous norm. The topology of $\mathrm{E}$ can then be defined by an increasing sequences $\left(\|\|_{k}\right)$ of norms (the index set is

$\mathrm{N}=\{1,2,3, \ldots \ldots . .\}$.$) . Let K_{k}$ denotes equipped with the norm $\|\cdot\|_{k}$ only and let $E_{k}$ be the completion of $E_{k}$. The identity mapping $E_{k+1} \rightarrow E_{k}$ has a unique extension $\phi_{k}: E_{k+1} \rightarrow E_{k}$ and this latter mapping is called canonical. The space $\mathrm{E}$ is said to be countably normed if the system $\left(\|\cdot\|_{k}\right)$ can be chosen in such a way that each $\phi_{k}$ is injective.

To give an example of a countably normed space,assume that $\mathrm{E}$ has an absolute basis i.e. there is a sequence $\left(X_{n}\right)$ in E such that every $\left(\xi_{n}\right)$ is a sequence of scalars. Then $\mathrm{E}$ is isomorphic to the Kothe sequence space

$\mathrm{K}(\mathrm{a})=\mathrm{K}\left(a_{n}^{k}\right)=\left\{\left(\xi_{n}\right)\left\|\left(\xi_{n}\right)\right\|_{k}=\sum_{n}\left|\xi_{n}\right| a_{n}^{k}\langle\infty \forall K\}\right.$

Where $a_{n}^{k}=\left\|X_{n}\right\|_{k}$. The topology of $\mathrm{K}(\mathrm{a})$ is defined by the norms ||$_{k}$. The completions

$\left(K(a)_{k}\right) \quad$ Can be isometric ally identified with ${ }^{\ell_{1}}$ and then the canonical mapping $\phi_{k}: \ell_{1} \rightarrow \ell_{1}$ is the diagonal transformation $\left(\xi_{n}\right)_{n} \rightarrow\left(\left(a_{n}^{k} / a_{n}^{k+1}\right) \xi_{n}\right)_{n}$ which is clearly injective. Therefore $\mathrm{E}$ is countably normed. Consider now a nuclear Frechet space $E$ which admits a continuous norm.The topology of E can be defined by a sequence $\left(\|.\|_{k}\right)$ of Hilbert norms, that is, $\|X\|_{k}=\langle X, X\rangle_{k} \mathrm{X} \in E$.where $\langle., .\rangle_{k}$ is an inner product of $\mathrm{E}$.

Theorem (1.1):

If a nuclear Frechet space $\mathrm{E}$ is countably normed, then the topology of $\mathrm{E}$ can be defined by a sequence of Hilbert norms such that the canonical mappings $\phi_{k}: E_{k+1} \rightarrow E_{k}$ are injective.

Suppose finally that $\left(\mathrm{X}_{n}\right)$ is a basis of E. Since $\left(\mathrm{X}_{n}\right)$ is necessarily absolute.E can be identified with a Kothe space $\mathrm{K}(\mathrm{a})$.By the Grothendieck-Pietch nuclearity criterion, for every $\mathrm{K}$ there is $\ell$ with $\left(\mathrm{a}_{n}^{k} / a_{n}^{\ell}\right) \in \ell_{1}$ .Conversely, if the matrix $\left(\mathrm{a}_{n}^{k}\right)$ with $0<\mathrm{a}_{n}^{k} \leq a_{n}^{k+1}$ satisfies this criterion ,then the Kothe space $\mathrm{K}(\mathrm{a})$ defined through(1) is a nuclear Frechet space with a continuous norm and the sequence of coordinate vectors constitutes a basis.In particular, $(\mathrm{s})=\mathrm{K}\left(\mathrm{n}^{k}\right)$.The topology of such a nuclear Kothe space can also defined by the sub-norms, $\left|\left(\xi_{n}\right)\right|_{k, \infty}=\sup _{n}\left|\xi_{n}\right| a_{n}^{k}$.

An Imbedding Theorem:

We are now ready to prove the following two conditions are equivalent: 
(1) E is countably normed,

(2) $\mathrm{E}$ is isomorphic to a subspace of a nuclear Kothe space which admits a continuous norm.

Moreover, the Kothe space in (2) can be chosen to be a quotient of (s).

Proofs:

As explained before, we know that a nuclear Kothe space with a continuous norm is countably normed. Since countably normedness is inherited by subspaces the implication $(2) \Rightarrow(1)$ is clear.

To prove $(1) \Rightarrow(2) \quad$ we choose a sequence $\left(\|\cdot\|_{k}\right)$ of Hilbert norms defining the topology of $E$ such that each canonical mapping $\phi_{k}: E_{k+1} \rightarrow E_{k}$ is injective (Theorem1).Let $\quad \mathrm{U}_{k}=\left\{\mathrm{X} \in E\|X\|_{k} \leq 1\right\}$ and identity $\left(\mathrm{E}_{k}\right)$ 'with

$\mathrm{E}_{k}^{\prime}=\left\{f \in \mathrm{E}\left|\|f\|_{k}^{\prime}=\sup \right|<x, f>\mid<\infty\right\}$

Then $\phi_{k}^{\prime}: \mathrm{E}_{k}^{\prime} \rightarrow E_{k+1}^{\prime}$ is simply the inclusion mapping. As a Hilbert space, $\mathrm{E}_{k+1}^{\prime}$ is reflexive. Using this and the fact that $\phi_{k} \quad: \mathrm{E}_{k+1} \rightarrow \mathrm{E}_{k}$ is injective ,one sees easily that $\phi_{k}^{\prime}\left(E_{k}^{\prime}\right)=E_{k}^{\prime}$ is dense in, $\mathrm{E}_{k+1}^{\prime}$.

We can construct in each $\mathrm{E}_{k}^{\prime}$ a sequence $\mathrm{f}_{n}^{(k)}$ of functional with the following properties:

$\mathrm{U}_{k}^{o} \subset\left\{\mathrm{f}_{n}^{(k)} \mathrm{n} \in N\right\}^{o o}$

$\left\{\mathrm{n}^{1} \mathrm{f}_{n}^{(k)} \mathrm{n} \in N\right\}$

equicontinuous for every $\ell$.

Now set $\mathrm{g}_{n}^{(1)}=\mathrm{f}_{n}^{(1)} \quad, \mathrm{n} \in \mathrm{N}$ and using the fact that $\mathrm{E}_{k}$ is dense in every $\mathrm{E}_{k}^{\prime}$ choose $\mathrm{g}_{n}^{(k)} \in E_{1}^{\prime}$,

$\geq 2, n \in N$ with

$$
\left\|f_{n}^{(k)}-g_{n}^{(k)}\right\|<2^{-n}
$$

In the construction of the desired Kothe space $\mathrm{K}(\mathrm{a})$ we will use two indices $\mathrm{k}$ and $\mathrm{n}$ to enumerate the coordinate basis vectors.

First,set

$\mathrm{a}_{k n}^{f}=2^{k} n^{2 \ell}, k, n \in N, \ell>k$

Then choose $\mathrm{a}_{k n}^{k}, a_{k n}^{k-1}, \ldots . a_{k n}^{1}$ so that

$1>a_{k n}^{k} \geq a_{k n}^{k-1} \geq \ldots . . \geq a_{k n}^{1}>0, k, n \in N$

$\frac{a_{k n}^{\ell+1}}{a_{k n}^{\ell+2}} \geq \frac{a_{k n}^{\ell}}{a_{k n}^{\ell+1}}, k, n \in N, \ell \leq k$

$\mathrm{a}^{\ell n} \leq \frac{1}{\left\|g_{n}^{(k)}\right\|_{\ell}^{\prime}}, k, n \in N, \ell \leq k$

Note that holds trivially for $\quad \ell>k$.Consequently, if $K\left(a_{k n}^{\ell}\right)=K(a)$ is nuclear, then it is also isomorphic to a quotient space of (s).But by for every $\ell \geq 2$

$$
\sum_{k=1}^{\infty} \sum_{n=1}^{\infty} \frac{a_{k n}^{\ell}}{a_{k n}^{\ell+1}}=\sum_{k=1}^{\ell-1} \sum_{n=1}^{\infty} \frac{a_{k n}^{\ell}}{a_{k n}^{\ell+1}}+\sum_{k=1}^{\infty} \sum_{n=1}^{\infty} \frac{a_{k n}^{\ell}}{a_{k n}^{\ell+1}} \leq \sum_{k=1}^{\infty} \sum_{n=1}^{\infty} \frac{a_{k n}^{\ell}}{a_{k n}^{\ell+1}}+\sum_{k=1}^{\infty} \sum_{n=1}^{\infty} \frac{a_{k n}^{k}}{a_{k n}^{k+1}}<(\ell-1) \sum_{n=1}^{\infty} \frac{1}{n^{2}}+\sum_{k=1}^{\infty} \sum_{n==1}^{\infty} \frac{1}{2^{k} n^{2(k+1)}}<\infty
$$

To imbed $\mathrm{E}$ into $\mathrm{K}(\mathrm{a})$ we set $\mathrm{Ax}=\left(\left\langle\mathrm{x}, \mathrm{g}_{n}^{(k)}\right\rangle\right), \mathrm{x} \in E$. We have to show that $\mathrm{Ax} \in K(a), \quad \mathrm{A}: \mathrm{E} \rightarrow \mathrm{K}(\mathrm{a})$ is a continuous injection and that $\mathrm{A}^{-1}: \mathrm{A}(\mathrm{E}) \rightarrow \mathrm{E}$ is also continuous.

Fix $\ell \geq 2$. Applying ( 3$)$ to the sequence $\left(\mathrm{f}_{n}^{(k)}\right)_{n}, \mathrm{k}=1 \ldots . . \ell-1$, we can find an index an index $\mathrm{p} \geq \ell$ and a constant $\mathrm{C}$ such that 
$\sup _{k<1, n} 2^{k} n^{2 \ell}\left|<X, f_{n}^{(k)}>\right| \leq C\|X\|_{p}, X \in E$

From (4), (5) and ( 8) we then get for every $X \in E$,

$$
\begin{aligned}
|A X|_{\ell, \infty} & =\sup _{k, n} a_{k n}^{\ell}\left|<X, g_{n}^{(k)}>\right| \leq \sup _{k<\ell, n} a_{k n}^{\ell}\left|<X, g_{n}^{(k)}>\right|+\sup _{k \geq \ell, n} a_{k n}^{\ell}\left|<X, g_{n}^{(k)}>\right| \\
& \leq \sup _{k, n} 2^{k} n^{2 \ell}\left|<X, g_{n}^{(k)}>\right|+\sup _{k \geq \ell, n} \frac{1}{\left\|g_{n}^{(k)}\right\|_{\ell}}\left|<X, g_{n}^{(k)}>\right| \\
& \leq \sup _{k<\ell, n} 2^{k} n^{2 \ell}\left\|g_{n}^{(k)}-f_{n}^{(k)}\right\|_{k}^{\prime}\|X\|_{k} \\
& +\sup _{k<\ell, n} 2^{k} n^{2 \ell}\left|<X, f_{n}^{(k)}>\right|+\|X\|_{\ell} \leq C^{\prime}\|X\|_{p^{\prime}}
\end{aligned}
$$

Where $C=\operatorname{Sup}_{n} n^{2 \ell} 2^{\ell-n}+C+1<\infty$

Consequently, $\mathrm{AX} \in \mathrm{K}(\mathrm{a})$ and $\mathrm{A}: \mathrm{E} \rightarrow \mathrm{k}(\mathrm{a})$ is continuous. From (2) it follows that for every $\mathrm{X} \in \mathrm{E}$.

$\|X\|_{\ell}=\sup _{f \in U_{\ell}^{o}}|<X, f>| \leq \sup _{n}\left|<X, f_{n}^{(\ell)}>\right|$

Further since $a_{\ell n}^{\ell+1}>1$

$$
\begin{aligned}
\sup _{n} \mid<X, f_{n}^{(\ell)} & >\left|\leq \sup _{n}\right|<X, f_{n}^{(\ell)}-g_{n}^{(\ell)}>\left|+\sup _{n}\right| X, g_{n}^{(\ell)} \mid \\
& \leq \sup _{n}\left\|f_{n}^{(\ell)}-g_{n}^{(\ell)}\right\|_{\ell}\|X\|_{\ell}+\sup _{k, n} a_{k n}^{\ell+1}\left|<X, g_{n}^{(k)}>\right| \\
& \leq \frac{1}{2}\|X\|_{\ell}+|A X|_{\ell+1, \infty} .
\end{aligned}
$$

Thus,by (9)and(10) we have for every $\mathrm{X} \in E$.

$$
\|X\|_{\ell} \leq|A X|_{\ell+1, \infty}
$$

Since ${ }^{\ell}$ is arbitrary, this shows that A is injective and that $A^{-1}: A(E) \rightarrow E$ is continuous.

Finally, we remark that is not possible to find a single nuclear Frechet space with basis and a continuous norm containing all countably normed nuclear spaces and subspaces.

\section{References}

[1] Adasch,N.: Topological vector spaces,lecture notes in maths, springer-verlag,1978.

[2] Grothendieck, A: Topological Vector spaces,Goedan and Breach,NewYork,1973.

[3] Holmstrom, L: A note on countably normed nuclear spaces, Pro. Amer. Math. Soc. 89(1983), p. 453.

[4] Litvinov, G.I.: Nuclear Space,Encyclopaedia of Mat Hematics, Kluwer Academic Publishers Spring-Verlag, 2001.

[5] Komura,T. and Y.Komura : Uber die Einbettung der nuclear Raume in (S) ${ }^{N}$, Mathe,Ann.162,1966, pp. 284-288. 